\title{
Graphene in Solar Cells
}

\section{Kal Renganathan Sharma*}

Department of Physics, College of Science and Technology, Texas Southern University, Houston, TX, USA

The Nobel Prize in Physics in the year 2010 was awarded to Profs. A.K. Geim and K. Novoselov for their research work on graphenes. Graphene has a two-dimensional lattice structure that is different from the 14 Bravais lattice structures instructed in the Materials Science courses. It has a honey-comb structure of benzene rings allowing for delocalization of electrons and unhindered electron transport. The graphene sheet is planar. Graphene possesses 200 times greater electron mobility compared with Silicon. In order to maintain the octet configuration, at a given juncture in time, some rows of benzene rings have three alternate single and double bonds, some rows have two double bonds and the rest single bonds in an alternating manner. I have authored a book "Graphene Nano materials", Momentum Press that is expected to be on the bookshelves in April 2014. Graphene market is expected to grow to a size of $\$ 675$ million dollars by the year 2020 allowing for a scalable model of graphene fabrication. International standards are yet to be developed for graphene. The cost of production of graphene is $\sim \$ 60$ per square inch on copper substrate. The cost of production needs to be reduced to less than $\sim \$ 1$ per square inch in order for some applications to be profitable. Expectations are high for graphene to be used in solar cells and towards the sun shot initiative of the department of energy. There are three ways graphene can be used in optical devices.

\section{AR, Anti-Reflection Coatings}

Studies have shown that over $10 \%$ increase in transmittance can be observed upon use of nanostructured coating on both sides of a glass slide compared with uncoated glass. Nanostructured silica is used as antireflection coating. For angle of incidences less than $70^{\circ}$ the transmittance of nanostructured coated glass was found to be about $99 \%$, up from $92 \%$ for broadband transmittance between 350 $\mathrm{nm}-1800 \mathrm{~nm}$. Less reflection is effected by the use of nanostructured coating. The incident optical beam when going from a rarer to denser medium refracts with an angle of refraction less than the angle of incidence. This would mean less reflection from the surface and more transmittance.It has been shown that when coatings with lower refractive indices are used Fresnel reflection can be virtually eliminated. Oblique angle $\mathrm{SiO}_{2}$ nanowires and Nano rods can be used to prepare advanced nanostructured antireflection coatings. These can be used in next generation sensors and optical windows. In this approach the reflection losses are minimized. AR coatings can be designed using a GA, genetic algorithm and fabricated using oblique angle deposition. Prof. Shubert's research group at Rensaellaer Polytechnic Institute, Troy, NY have developed an analytical model that can be used to predict the porosity and deposition rate of nanoporous films grown by obliqueangle deposition.

Refractive index of the coating is an important parameter in the design of solar cells and other optical devices where the coating is applied. Materials with $\mathrm{n}$, refractive index in the range of 1-1.4 are limited. Aerogels may foot the bill but the control of its thickness is difficult. Prof. F. Schubert's research group deposit silica Nano rods by evaporation. They obtain good control of refractive index by varying the angle of incidence. They obtain a material with refractive index of 1.1 for an incident angle of $80^{\circ}$, material with $\mathrm{n}=1.07$ at an incident angle of $85^{\circ}$. A world record for material with low $n=1.05$ was obtained at an incident angle of $87^{\circ}$. They conduct research in development of graded-index AR, anti-reflection coatings for a new class of optical materials. Standard AR coating is $0.25 \lambda$ thick and zero reflectivity is seen at one wavelength. This can be used in some applications. The electromagnetic spectrum from the sun is broader in scope, ranging from UV, ultraviolet and IR, infra-red.

The principle of total internal reflection is used to obtain zero transmission losses. When optical ray travels from a denser to rarer medium the angle of refraction will be greater than the angle of incidence. At some higher angle of incidence the angle of refraction would be greater than $90^{\circ}$. This would mean the ray that was to be refracted has been totally internally reflected. They deposit five layers of decreasing refractive indices. The difference in refractive index between two adjacent layers is small. The output efficiency of LEDs can be improved by use of AR coating. Another application is in the area of super luminescent diodes. High reflectance mirrors and Bragg reflectors can use AR coating. Fresnel reflection can be eliminated from an interface of AIN/air, aluminum nitride.

\section{SG used for Greater Electron Mobility}

Electrons and holes are allowed to recombine in SG to form photons. More electron mobility allowed in the single layer graphene sheet leads to more electric current and hence more light to electricity conversion efficiency can be expected. The operations of LEDS, light emitting diodes are based on electro-photoluminscence phenomena. Light is emitted from a semi-conductor material when current or voltage is applied. LEDs prepared using p-type graphene doped with a $\mathrm{p}$ type dopant and $\mathrm{n}$ type graphene doped with $\mathrm{n}$ type dopant was patented by Samsung LEDs Co., S. Korea, Graphene superlattice with graphene Nano ribbons is used to connect the p-type and n-type graphene. The graphene nanoribbon is zig-zag shaped on opposite edges. The width of the nanoribbon is $3-20 \mathrm{~nm}$. The interval between adjacent Nano ribbons is $2-15 \mathrm{~nm}$. The graphene supperlattice has periodically arranged quantum dot patterns. The quantum dots can have the same size/shape of may be allowed to differ in size/shape. Depending on the size the quantum dots may correspond to red, green and blue photolumuniscences. $\mathrm{p}$ type dopants may be oxygen, gold or bismuth atoms or acidic compounds. The concentration level is about $10^{-20} \mathrm{~cm}$ ${ }^{2}-10^{-5} \mathrm{~cm}^{-2} . \mathrm{n}$ type dopants can be nitrogen, fluorine, manganese atoms or ammonia. Concentration of the dopant is between $10^{-20} \mathrm{~cm}^{-2}-10^{-5}$ $\mathrm{cm}^{-2}$. The p-type dopant and n-type dopant may be spaced apart at constant distances. When voltage is applied to the p-type graphene and

*Corresponding author: Kal Renganathan Sharma, Department of Physics, College of Science and Technology, Texas Southern University, Houston, TX, USA Tel: 281-256-2976; E-mail:jyoti_kalpika@yahoo.com

Received February 18, 2014; Accepted February 18, 2014; Published February 24, 2014

Citation: Sharma KR (2014) Graphene in Solar Cells. J Laser Opt Photonics 1: e101. doi:10.4172/2469-410X.1000e101

Copyright: (c) 2014 Sharma KR. This is an open-access article distributed under the terms of the Creative Commons Attribution License, which permits unrestricted use, distribution, and reproduction in any medium, provided the original author and source are credited. 
$\mathrm{n}$-type graphene in the forward direction holes in the p-type graphene and electrons in the n-type graphene move toward the active graphene. Energy bandgap with the dopant can be seen for both p-type and n-type graphene. Electrons and holes recombine in active graphene supperlattice and emit photons. Light corresponding to the bandgap is emitted. The higher electron mobility in graphenes translated into high brightness emission.

Graphene field effect transistor was studied for its distinct electrical characteristics of a p-type and n-type graphene used in LEDs. The current-voltage characteristics (I vs. V) of the photoluminescent device can be seen to be non-linear and deviates from the predictions of Ohm's law of electricity. Dirac voltage can be seen at low currents and a concave curvature in the curve in the electron flow region ( $p$ doping) can be seen. The curve is a mirror reflection of the electron flow region in the whole flow ( $\mathrm{n}$ doping) region of the graph.

Graphene quantum dots can have the same diameter and may be dispersed between p-type graphene and the $\mathrm{n}$-type graphene. The size range of quantum dots is $1-100 \mathrm{~nm}$. The size of the quantum dots can be directly related to the photoluminescence wavelength. Longer photolumiscence wavelengths can be seen from larger quantum dots. The photoluminescence characteristics such as mono color or dichromatic or multi-color can be controlled by controlling the size of the quantum dots.
When the LED is made from compound semiconductor the composition of the compound can be changed in order to control the output photoluminiscent characteristics. By attaching a functional group to the quantum dot the band gap or doping characteristics of the LED can be changed. When a voltage is applied to the p-type graphene and the n-type graphene holes and electrons move toward the active graphene. Electrons and holes recombine and a photon is emitted corresponding to the energy band gap. Photolumiscence caused by quantum dots of the active graphene may result in amplification of light generated due to the recombination of holes and electrons. Due to the higher mobility in graphene a higher level of current may be supplied in order to achieve high brightness emissions.

\section{Energy Storage}

NGP, nanographene platelets can be used in energy storage applications. Graphene can be used in rechargeable batteries, 50 billion cell phones, laptops, tablets are expected to be in operation in the next decade. Graphene can be used to make higher capacity electrodes. Triple junction solar cells with light to power conversion efficiency of $41 \%$ have been reported by Siemens and Semprius Inc. A three spectrum solar cell is possible by manipulation of the size of the quantum dots in doped graphene. Polyacetylene can be converted to graphene and a superconductor can be realized at certain temperatures. Alkali doped graphenes have potential to make good superconductors with higher transition temperatures. 